\title{
Effectiveness of a telephone delivered and a face-to- face delivered counseling intervention for smoking cessation in patients with coronary heart disease
}

Citation for published version (APA):

Berndt, N., Bolman, C., Froelicher, E. S., Mudde, A., Candel, M., de Vries, H., \& Lechner, L. (2014). Effectiveness of a telephone delivered and a face-to-face delivered counseling intervention for smoking cessation in patients with coronary heart disease: a 6-month follow-up. Journal of Behavioral Medicine, 37(4), 709-724. https://doi.org/10.1007/s10865-013-9522-9

Document status and date:

Published: 01/08/2014

DOI:

10.1007/s10865-013-9522-9

Document Version:

Publisher's PDF, also known as Version of record

Document license:

Taverne

Please check the document version of this publication:

- A submitted manuscript is the version of the article upon submission and before peer-review. There can be important differences between the submitted version and the official published version of record.

People interested in the research are advised to contact the author for the final version of the publication, or visit the DOI to the publisher's website.

- The final author version and the galley proof are versions of the publication after peer review.

- The final published version features the final layout of the paper including the volume, issue and page numbers.

Link to publication

\footnotetext{
General rights rights.

- You may freely distribute the URL identifying the publication in the public portal. please follow below link for the End User Agreement:

www.umlib.nl/taverne-license

Take down policy

If you believe that this document breaches copyright please contact us at:

repository@maastrichtuniversity.nl

providing details and we will investigate your claim.
}

Copyright and moral rights for the publications made accessible in the public portal are retained by the authors and/or other copyright owners and it is a condition of accessing publications that users recognise and abide by the legal requirements associated with these

- Users may download and print one copy of any publication from the public portal for the purpose of private study or research.

- You may not further distribute the material or use it for any profit-making activity or commercial gain

If the publication is distributed under the terms of Article 25fa of the Dutch Copyright Act, indicated by the "Taverne" license above, 


\title{
Effectiveness of a telephone delivered and a face-to-face delivered counseling intervention for smoking cessation in patients with coronary heart disease: a 6-month follow-up
}

\author{
Nadine Berndt • Catherine Bolman • Erika Sivarajan Froelicher • \\ Aart Mudde • Math Candel • Hein de Vries • \\ Lilian Lechner
}

Received: October 18, 2012 / Accepted: May 29, 2013/Published online: June 13, 2013

(C) Springer Science+Business Media New York 2013

\begin{abstract}
Smoking cessation interventions for cardiac patients need improvement given their weak effects on long-term abstinence rates and low compliance by nurses to implementation. This study tested the effectiveness of two smoking cessation interventions against usual care in cardiac patients, and conditional effects for patients' motivation to quit and socio-economic status (SES). An experimental study was conducted from 2009 to 2012 for which Dutch cardiac patient smokers were assigned to: usual care (UC; $n=245$ ), telephone counseling (TC; $n=223$ ) or face-to-face counseling (FC; $n=157)$. The three groups were comparable at baseline and had smoked on average 21 cigarettes a day before hospitalization. After six months, interviews occurred to assess self-reported
\end{abstract}

N. Berndt $(\bowtie) \cdot$ C. Bolman · A. Mudde .

L. Lechner

Department of Psychology, Open University of the Netherlands,

Valkenburgerweg 177, PO Box 2960, 6401 DL Heerlen,

The Netherlands

e-mail: nadine.berndt@ou.nl

E. S. Froelicher

Department of Physiological Nursing, School of Nursing,

University of California, San Francisco, CA, USA

E. S. Froelicher

Department of Epidemiology and Biostatistics, School of

Medicine, University of California, San Francisco, CA, USA

M. Candel

Department of Methodology and Statistics, School for Public Health and Primary Care, Maastricht University, Maastricht,

The Netherlands

H. de Vries

Department of Health Promotion, School for Public Health

and Primary Care, Maastricht University, Maastricht,

The Netherlands smoking status. Patients in the TC and FC group had significantly higher smoking abstinence rates than patients in the UC group ( $p \leq 0.05$ at all times). Regression analysis further revealed significant conditional effects of the interventions on smoking abstinence in patients with lower SES, with a larger effect for TC than FC when compared to UC. These findings suggest that intensive counseling is effective in increasing short-term abstinence rates, particularly in patients with lower SES. Future studies need to investigate how patients with higher SES can profit equally from these type of interventions.

Keywords Coronary heart disease - Smoking cessation . Telephone counseling · Face-to-face counseling . Socio-economic status · Effectiveness study

\section{Background}

Smoking cessation is the single most effective action for secondary prevention of coronary heart disease (CHD). It significantly reduces the risk of mortality, re-hospitalization and reoccurrence after a coronary event (Critchley \& Capewell, 2003; Mohiuddin et al., 2007; Van Spall et al., 2007). Nevertheless, more than $50 \%$ of smokers with CHD continue to smoke after hospital discharge (Rigotti et al., 2012; Scholte op Reimer et al., 2006). Continued smoking in CHD patients has been related to high nicotine dependence levels, low intentions to quit (Berndt et al., 2012b; Costa et al., 2010) and insufficient cessation support (Barth et al., 2006; Bolman et al., 2002b; Rigotti et al., 2008). A nurse-led minimal intervention strategy at the bedside of cardiac patients, to help smokers to quit, was evaluated and subsequently implemented on a large scale in Dutch cardiac wards. This intervention, although 
effective in the short term (Bolman et al., 2002c), only had a minimal impact on public health due to implementation difficulties in practice (Bolman et al., 2002a; Segaar et al., 2006, 2007). Barriers to adequate implementation of smoking cessation interventions in hospital units include lack of time, difficulties in providing care after patient discharge, and competing priorities (Hennrikus et al., 2005; Kotz et al., 2008; Sarna et al., 2009; Wetta-Hall et al., 2005). Due to the lack of suitable interventions for nursing practice, many patients continue to smoke, resulting in high risks of recurrent cardiovascular disease and death (Critchley \& Capewell, 2003; Gerber et al., 2009). The delivery of effective smoking cessation interventions thus remains a priority public health issue.

Hospital-initiated interventions currently are the gold standard for smoking cessation for patients with CHD (Rigotti, 2009; Smith \& Burgess, 2009b). In this sense, evidence-based guidelines recommend combined approaches that use behavioral and pharmacological therapies (Fiore et al., 2008; Van Weel et al., 2005) since these have shown to be most likely to increase cessation rates (Rigotti et al., 2012). Moreover, to overcome the shortcomings of bedside counseling in hospitals, guidelines recommend approaches such as the ' 5 A's', also simplified to the AskAdvise-Refer strategy (Bentz et al., 2007; Orleans et al., 2006). The Ask-Advise-Refer strategy emphasizes a shift in smoking cessation treatment from the inpatient to the outpatient setting, thus enabling ward nurses to refer patients to competent community health professionals dedicated to smoking cessation counseling with adequate time (Berndt et al., 2011).

Two intensive smoking cessation behavioral interventions; telephone counseling (TC) and face-to-face counseling (FC), have been shown to be equally effective in enhancing cessation rates in the general population (Lancaster \& Stead, 2005; Rigotti et al., 2008; Stead et al., 2006). Smith and Burgess showed that TC might achieve higher success rates in cardiac patients when it is initiated during hospital admission with bedside counseling, and includes several sessions during a follow-up period of two months after discharge (Smith \& Burgess, 2009a, b). Their study built on a classic study from 1990 testing the efficacy of a nurse-managed program for cardiac inpatients motivated to quit, which yielded cessation rates in the experimental group that were twice as high as in the control group (Taylor et al., 1990). A comparable intervention was used in women with cardiovascular disease, revealing high rates of sustained abstinence (Froelicher et al., 2004). In sum, high intensity interventions for cardiac patients have yielded significantly better cessation rates than low intensity interventions (Huttunen-Lenz et al., 2010; Rigotti et al., 2012).

Despite the accumulating evidence of the effectiveness of intensive interventions, cardiac patients that are current smokers are still not offered the optimal smoking cessation treatment (Freund et al., 2008; Van Spall et al., 2007; Rigotti, 2009). From the above named studies it becomes apparent that in order to reach highest effectiveness, interventions should be initiated at hospital admission, include a quit advice from the patient's physician in charge (Kotz et al., 2007), be continued for at least one month after discharge, and consist of numerous contacts (Barth et al., 2008; Fiore et al., 2008; Rigotti et al., 2012). Moreover, nicotine replacement therapy (NRT) is an optimal adjunct to behavioral counseling. NRT has been shown to be both safe and effective for patients with cardiovascular disease (Benowitz \& Gourlay, 1997; Reid et al., 2006; Van Berkel et al., 1999), while it suppresses withdrawal symptoms and can be prescribed for most patients (Boyle et al., 2005; Reid et al., 2006; Rigotti et al., 1999; Hughes et al., 2007).

While studies have investigated the effectiveness of counseling methods, few have examined the effectiveness of combined approaches using intensive behavioral counseling and NRT in patients with established CHD. Furthermore, to our knowledge, no previous studies have compared the effectiveness of different delivery methods of smoking cessation counseling combined with NRT in cardiac patients, nor have the effects of the types of counseling (TC vs. FC) been studied. However, identifying which intervention is most effective for specific subgroups of patients with CHD can provide important recommendations for future interventions. The main benefits of smoking cessation counseling provided by telephone are that it is easy accessible, time-saving, and involves low costs. TC can be delivered outside the cardiac ward and be scheduled in response to the specific characteristics and needs of the patient, making it a convenient approach for providing smoking cessation counseling to those patients without mobility or those who are resistant to FC (Lichtenstein et al., 1996; Reese et al., 2006). A review on the effectiveness of TC for smoking cessation exploring the effect of motivation did not find a significant difference in the effect size between those studies that specifically enrolled smokers motivated to quit, and those that did not include smokers on the basis of motivation. The authors assumed that relatively high proportions of smokers may have been interested in quitting and concluded that proactive TC, in which the counselor initiates calls to provide smoking cessation support, is particularly beneficial for smokers interested in quitting (Stead et al., 2006). Hence, it is conceivable that patients with high quit motivation may profit in particular from TC when compared to those with low quit motivation. In this regard, high socio-economic status (SES) groups are recognized as more successful in quitting due to strong motivations (Fernandez et al., 2006), and thus may also profit more from TC than low SES 
groups. Previous studies have indicated that low SES groups are more likely to have higher CHD rates and smoking prevalence than high SES groups (Ramsay et al., 2009), and are less likely to quit smoking successfully (Hiscock et al., 2011; Reid et al., 2010). It is thus conceivable that patients with lower SES, often less motivated to quit, benefit particularly from more intensive interventions (Siahpush et al., 2006), as previously indicated by a Dutch study testing the effectiveness of FC in a general population of smokers (Van Emst, 2007).

The objectives of this study were to (a) examine the effectiveness of a telephone delivered and a face-to-face delivered smoking cessation intervention using NRT on cardiac patients' smoking behavior six months after hospital discharge by comparing each intervention to usual care, and (b) test conditional effects of the interventions on smoking abstinence for patients varying in their motivation to quit, and for low versus high SES groups.

\section{Method}

\section{Design}

An experimental study design with sequential cross-over randomization at the ward level was used (see Fig. 1). After completion of care as usual (UC), the cardiac wards were randomized to either implement TC or FC. Four of the cardiac wards were allocated to implement TC, and the other four wards were allocated to implement FC. After completion of the intervention per cardiac ward, there was a one month wash-out period. Then, each cardiac ward implemented the other intervention. Those wards who first implemented TC subsequently implemented FC, whereas the other four cardiac wards implemented the interventions in reverse order. The current design was chosen to overcome problems related to traditional randomization such as learning effects by the nursing staff, as well as contamination between patients.

\section{Setting}

Forty-six cardiac wards in urban, leading clinical and academic hospitals throughout the Netherlands were approached to assess whether they met the inclusion criteria. Wards were included if they provided cardiac nursing care, did not offer any form of smoking cessation intervention to cardiac patients at the bedside, if there was no outpatient smoking cessation department available at the hospital, and if they had an adequate patient stream to ensure sufficient patients for inclusion. Intensive care units, intermediate care units, and combined heart and lung unit were excluded to guarantee consistency. Of the 46 wards that were approached, eight met all the inclusion criteria and were invited to participate.

The Medical Research Ethics Committee (MERC) of the VU Medical Center Amsterdam approved the study protocol. Subsequently, the MERCs and/or Board of Directors of each hospital endorsed execution of the study. The study was registered with the Trial Registration (NTR2144).

Cardiac wards 1 to 4 :

\begin{tabular}{|c|c|c|c|c|}
\hline $\mathrm{T}_{0}$ & UC & $T_{1}$ & & \\
\hline & $\mathbf{T}_{0}$ & TC & $\mathrm{T}_{1}$ & \\
\hline & & $T_{0}$ & FC & $T_{1}$ \\
\hline
\end{tabular}

Cardiac wards 5 to 8 :

\begin{tabular}{|c|c|c|c|c|}
\hline $\mathrm{T}_{0}$ & UC & $T_{1}$ & & \\
\hline & $T_{0}$ & FC & $\mathbf{T}_{1}$ & \\
\hline & & $T_{0}$ & TC & $T_{1}$ \\
\hline
\end{tabular}

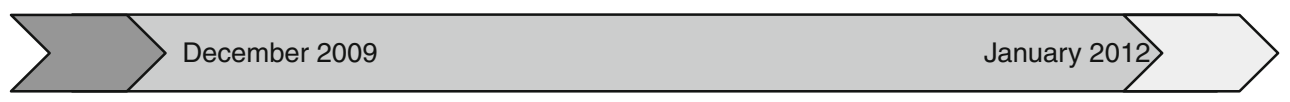

Fig. 1 Schematic view of the study design. $\mathrm{T}_{0}=$ baseline measurement with questionnaire; $\mathrm{UC}=$ usual care; TC = telephone counseling intervention; $\mathrm{FC}$ = face-to-face counseling intervention; $\mathrm{T}_{1}=$ six months follow-up measurement by telephone interview 
Recruitment and enrollment of patients

Ward nurses and cardiologists received a written stepwise protocol of the study with a flowchart and instructions for referral, and oral instructions about the inclusion process of eligible cardiac patients. Nurses were instructed to ask every eligible patient and to register data on those eligible patients not enrolled in the study. Inclusion criteria for patients were: smoking on average $\geq$ five cigarettes per day in the month prior to admission or, if not smoking, having quit smoking less than four weeks prior to admission; being $\geq 18$ years of age; being admitted to the cardiac ward for less than 96 hours and being hospitalized because of an acute coronary syndrome (ACS), stable angina, or other forms of chronic and acute heart diseases following the standards of the ICD-10 (World Health Organization, 2007). Patients who quit smoking in the four weeks prior to admission were also included because many patients already start feeling ill before hospital admission, and quit smoking in advance. They were considered as recent quitters with a high risk for relapse. Exclusion criteria were Dutch language limitations that would impede completion of the self-administered questionnaire, not owing a telephone at home or a mobile phone, a medically unstable cardiac situation or cognitive impairment.

Over an 18-month period beginning in December 2009, ward nurses approached eligible patients at the bedside, provided them with the necessary information about the study, and invited them to take part. If patients agreed to take part in the study, the nurses obtained written informed consent. In total, 245 cardiac patients were allocated to the UC group, 223 to the TC group, and 157 to the FC group. After six months, follow-up data was provided by 489 patients $(78.2 \%)$. In total, 196 patients completed the follow-up measurement in the UC $(80 \%), 170$ in the TC (76.2\%) and 123 in the FC group (78.3\%) (see Fig. 2).

A sample size calculation was conducted that assumed an $\alpha=0.05$ and $\beta=0.02$ with $1-\beta=0.80$ power, a medium effect size of $60 \%$ quitters in the experimental groups against $43 \%$ quitters in the UC group at 6-month follow-up, a $15 \%$ attrition rate, and adjustment for potential baseline confounding variables by which the sample size was reduced by $30 \%$. According to this calculation, 155 patients per group needed to be recruited, totalling 465 patients for the three groups. It was attempted to recruit more patients in order to increase statistical power in each of the three groups.

Protocol for experimental interventions

The details of the intervention protocols have been previously reported (Berndt et al., 2012a). In brief:
Usual care (UC)

All patients received standard in-hospital treatment for smoking cessation that consisted of an assessment of their smoking behavior, the delivery of brief quit advice and occasionally the delivery of an informational brochure. Quit advice was largely provided by cardiologists (and occasionally by ward nurses) while patients were on the cardiac ward.

Face-to-face counseling (FC) plus nicotine replacement therapy (NRT)

Patients in the FC group received UC, plus the combination therapy of FC with NRT. Nurses on cardiac wards followed the Ask-Advice-Refer strategy (Berndt et al., 2011; Orleans et al., 2006) in which patient smoking behavior was first assessed. Secondly, patients who were smokers were advised to quit, and thirdly, they were referred to outpatients' professional smoking cessation counselors. The counselors were sixteen nurses (others than the nurses on the cardiac ward) who followed a four day training course prior to the start of the study in providing FC. FC started within one week of patient recruitment and lasted three months, consisting of six face-to-face sessions of 30-45 $\mathrm{min}$ and ending with a follow-up call five weeks after the last session. The counselors worked with a protocol based upon the Transtheoretical Model (Prochaska \& DiClemente, 1983) which posits that behavior change involves progress through five stages. During each session the counselors discussed relevant themes and focused on determinants of smoking cessation and relapse important for each stage such as selfefficacy and smoking-related attitudes (see Fig. 3).

Ward nurses also provided nicotine patches to eligible patients with an information sheet on their necessity and instructions for usage. Cardiologists from participating cardiac wards agreed with the prescription of transdermal nicotine patches for cardiac patients. Eligibility for NRT was that there were no contra-indications and that the patient had smoked more than ten cigarettes a day before hospital admission. If eligible, the patient received the patches for eight weeks at no cost. The dosage was as follows: $21 \mathrm{mg} / 14 \mathrm{mg} / 7 \mathrm{mg}$ for patients who had smoked more than 20 cigarettes and 14/7 mg for patients who had smoked between ten and 20 cigarettes a day.

\section{Telephone counseling (TC) plus nicotine replacement therapy (NRT)}

TC started within one week of patient enrollment and had a structure and content highly comparable to FC (see Fig. 3). The main differences between the counseling methods were 


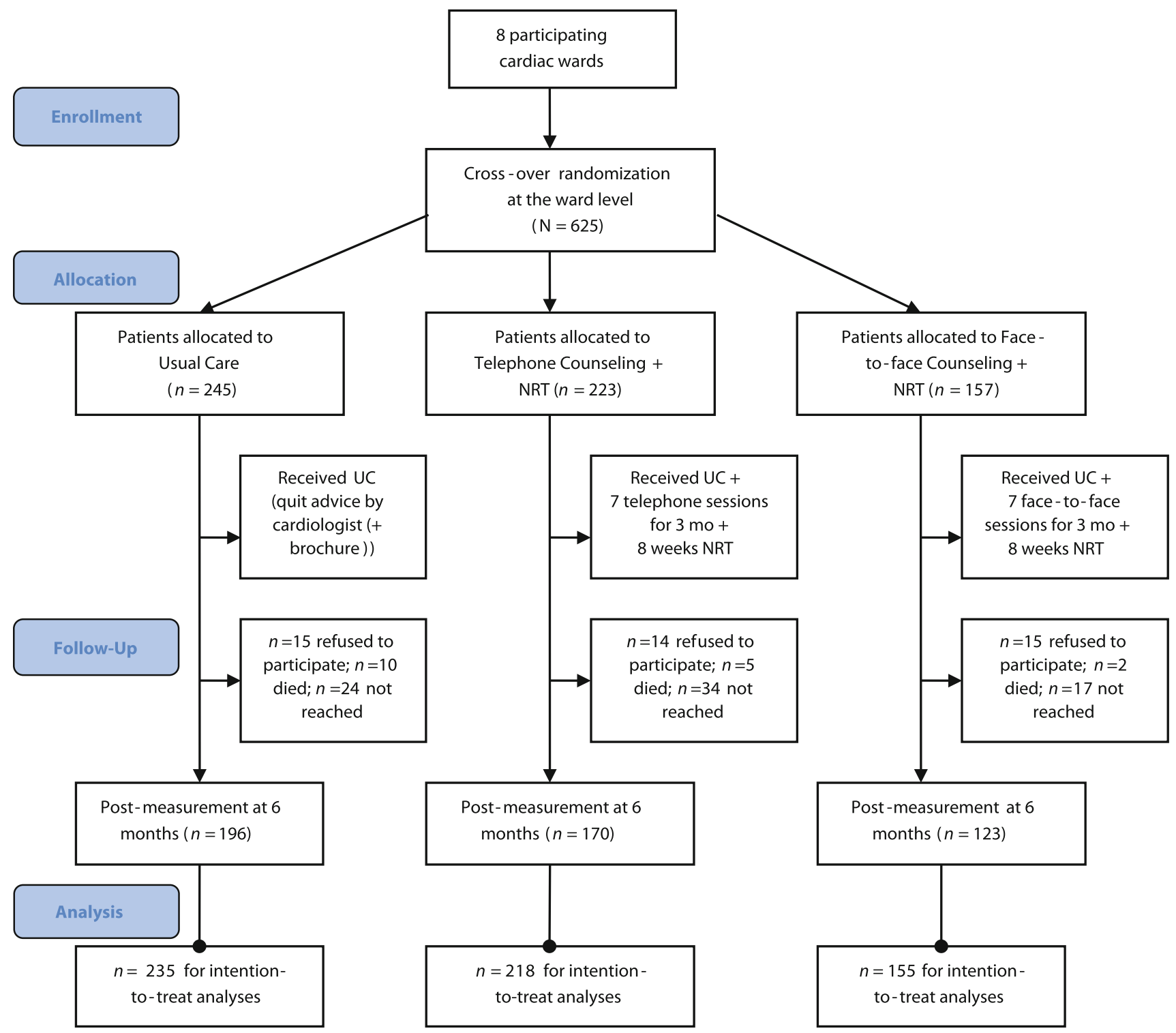

Fig. 2 Flow diagram of the experimental study testing the effectiveness of two intensive smoking cessation interventions in cardiac patients. $\mathrm{UC}=$ usual care; NRT = nicotine replacement therapy, in this trial nicotine patches

the provider and delivery method (face-to-face vs. telephone) and duration of the counseling. TC lasted for three months and consisted of seven telephone calls of $15 \mathrm{~min}$ duration. Due to its modality, TC allowed for shorter and more concise sessions than FC (patients were available for direct counseling and small-talks could be easily avoided). The Ask-Advice-Refer strategy as applied by ward nurses and the delivery of the nicotine patches to eligible patients were equivalent to that used in FC. Patients in both the FC and TC group got a new out-patient provider for delivering the interventions, the counseling for TC, however, was provided by four professional telephone counselors from the Dutch Expert Centre for Tobacco Control.
Data collection at baseline $\left(\mathrm{T}_{0}\right)$

At baseline $\left(\mathrm{T}_{0}\right)$ nurses administered a written questionnaire to patients and recorded relevant patient data. Nurses sent these documents monthly to the researchers. $\mathrm{T}_{0}$ included variables related to smoking cessation among patients with established CHD, i.e. demographic characteristics, clinical data and cardiac risk factors, smoking history and smoking patterns, psychological factors, and social cognitive factors. Variables were based on the I-Change Model (De Vries et al., 2005) and were found to be reliable in previous studies of Dutch cardiac patients (Van Berkel et al., 2000; Bolman \& de Vries, 1998). Data 


\begin{tabular}{|c|c|c|c|c|c|c|}
\hline \multicolumn{7}{|c|}{ Process of smoking cessation } \\
\hline \multicolumn{2}{|l|}{ Preparation } & \multicolumn{2}{|l|}{ Action } & \multicolumn{3}{|c|}{ Maintaintance } \\
\hline session 1 & session 2 & session 3 & session 4 & session 5 & session 6 & session 7 \\
\hline $\begin{array}{l}\text { - Striking a } \\
\text { balance } \\
\text { - Preparation } \\
\text { of cessation }\end{array}$ & $\begin{array}{l}\text { - Making a } \\
\text { decision } \\
\text { - Withdrawal } \\
\text { symptoms }\end{array}$ & $\begin{array}{l}\text { - The desire } \\
\text { of smoking }\end{array}$ & $\begin{array}{l}\text { - High risk } \\
\text { situations }\end{array}$ & $\begin{array}{l}\text { - Relapse } \\
\text { prevention }\end{array}$ & $\begin{array}{l}\text { - Theme } \\
\text { selection }\end{array}$ & $\begin{array}{l}\text { - Follow-up by } \\
\text { telephone }\end{array}$ \\
\hline \multicolumn{7}{|c|}{$\begin{array}{l}\text { Strategies: Motivational interviewing, self-control training, self-efficacy enhancement and relapse prevention. } \\
\text { Tailoring: Adapt content to the situation and need of the patient; retrospectively discuss pre-action stages }{ }^{b} \text {. }\end{array}$} \\
\hline week 1 & week 2 & week 3 & week 4 & week 5 & week 7 & week 12 \\
\hline TC: $15 \mathrm{~min}$ & TC: $15^{\prime}$ & TC: $15^{\prime}$ & TC: $15^{\prime}$ & TC: $15^{\prime}$ & TC: $15^{\prime}$ & TC: $15^{\prime}$ \\
\hline FC: $45 \mathrm{~min}$ & FC: $45^{\prime}$ & FC: $45^{\prime}$ & FC: $45^{\prime}$ & FC: $45^{\prime}$ & FC: $45^{\prime}$ & FC: $15^{\prime}$ \\
\hline
\end{tabular}

Fig. 3 Protocol of the TC and FC intervention. ${ }^{a}$ For TC, the themes of sessions 5 and 6 were discussed vice-versa. ${ }^{\mathrm{b}}$ Many patients already reside in the action stage because they quit at the time of hospital admission

from the assessments was obtained identically from all patients, disregarding their smoking status.

Baseline assessment of demographic data from each patient included sex, age, marital status, and SES (Mudde et al., 2006). SES included education level and was categorized as primary education (1) including primary schools and basic vocational schools; intermediate education (2) including secondary vocational schools and high school degrees; or tertiary education (3) including higher vocational school degrees, college or university degrees.

Cardiac risk factors included disease diagnosis and previous hospital admission in the past six months. Diagnosis was categorized as ACS (1), stable angina (2) and other forms of cardiac diagnosis (3).

The smoking history evaluation included nicotine dependence level, past smoking habits and current smoking. The six item Fagerström test was used to test for level of nicotine dependence (Heatherton et al., 1991). Past smoking habits were assessed by asking patients how many cigarettes they smoked on average per day and whether they had engaged in a 24-hour attempt to quit over the past 12 months (Mudde et al., 2006). Smoking behavior at admission was measured by asking patients whether they had been abstinent from smoking the past seven days $(0=$ yes; $1=$ no $)$.

The 14-item Hospital Anxiety and Depression Scale (HADS) was used to detect levels of depression and anxiety (Zigmond \& Snaith, 1983). The depression subscale was comprised of seven items $(\alpha=0.81)$ and included questions such as: "Do you take as much interest in things as you used to?". Response options ranged from 'very much/often' (0) to 'almost never' (3). The anxiety subscale also consisted of seven items $(\alpha=0.79)$ and asked "whether patients felt tense or whether they worried a lot" with answers ranging from 'not at all' (0) to 'very much' (3).

Self-efficacy to smoking abstinence was assessed using ten items related to the likelihood of being able to refrain from smoking in various situations (Dijkstra et al., 1996; Hoving et al., 2006). Answers were given on a five-point Likert scale ranging from 'definitely not' (0) to 'definitely yes' (4). An example of self-efficacy was: "Are you able to refrain from smoking when someone offers you a cigarette?" A self-efficacy score was constructed by averaging responses to the ten items $(\alpha=0.93)$, with higher scores signifying greater perceived self-efficacy in refraining from smoking.

Social modeling by the patients' partner (if applicable) was also assessed (Bolman \& de Vries, 1998; Mudde et al., 2006) with a single item which identified whether the patients' partner smoked $(0=$ smoking partner; $1=$ nonsmoking partner/no partner).

To measure intrinsic motivation to quit, three items were used based on an existing questionnaire (van Stralen et al., 2011) in line with self-determination theory (Ryan \& Deci, 2000). This measure was adapted for cardiac patients. An example was: "I believe it is worth making an effort to quit smoking" or "I am committed to my plan to quit smoking" with response options ranging from 'definitely not' (0) to 'definitely yes' (4). An intrinsic motivation score was calculated by averaging the responses to those items $(\alpha=0.83)$. Independent of the smoking status of the patient at hospital admission, intrinsic motivation was measured equally in all patients. 
For assessing intention to (remain) quit (Ajzen, 2002; Fishbein \& Ajzen, 2010), patients were asked how strongly they intended to quit smoking or intended to remain quit after hospital discharge, and how likely it was that they would refrain from smoking after hospital discharge on a five-point Likert scale ranging from 'not likely' (1) to 'very likely' (5) $(\alpha=0.86)$.

Since patients were enrolled over an 18-month period, and the study design used was likely to induce time effects, the total period of inclusion was subdivided into five categories (1 = Dec 2009-Jan 2010; 2 = Feb-June 2010; 3 = JulyNov 2010; 4 = Dec 2010-Jan 2011; 5 = Feb-June 2011) and included as dummy variables in relevant analysis.

Data collection at 6-month follow-up $\left(\mathrm{T}_{1}\right)$

At $T_{1}$ patients were interviewed by telephone by members of a professional call agency to obtain data about selfreported smoking abstinence, for which patients were reminded by mail. Telephone interviews were conducted to ensure blinding of outcome data collection and to decrease the likelihood of attrition. Two separate outcome measures were obtained:

Continued abstinence ( $C A$ ) was the primary outcome measure because it is regarded as a conservative measure in the assessment of smoking cessation (Hughes et al., 2003; Velicer \& Prochaska, 2004). CA was defined as being abstinent from smoking for at least 90 days $(0=n o$; $1=$ yes). For this purpose, patients were asked to specify their quit date at $\mathrm{T}_{1}$, or if they could not remember their exact quit date, the number of months or weeks they had been abstinent from smoking. Subsequently, the selfreported quit date was subtracted from the date of the telephone interview at $\mathrm{T}_{1}$. A 90-day period of CA was chosen because the interventions lasted for three months, and to be classified as CA patients had to be abstinent from smoking since the end of the last counseling session. The self-report of CA allowed patients to have smoked a maximum of five cigarettes in total over the whole period following their quit date (West et al., 2005).

7-day Point-Prevalence Smoking Abstinence (7-day $P P A)$ is considered to be a valid measure of smoking cessation (Velicer \& Prochaska, 2004) and was additionally used to obtain patients' self-reported smoking behavior over the previous seven days at $\mathrm{T}_{1}$ : 'Have you refrained from smoking during the past seven days, and not smoked even one puff?' $(0=$ no; $1=$ yes $)$.

Data management and analyses

Data was analyzed with SPSS version 19 and assessed for the range of values, outliers, missing values and normality. For the continuous scale variables, missing item scores for a patient were replaced with the average of the available item scores if less than $33 \%$ of the items had missing values. For analysis including follow-up data, 17 patients were missing because they died.

ANOVAs and $\chi^{2}$ tests were first applied to test for baseline differences in patient characteristics among the three groups. An attrition analysis was then conducted in order to determine possible selective loss at follow-up by means of a logistic regression analysis, using dropout $(0=n o ; 1=y e s)$ as the outcome and baseline characteristics and group assignment as predictive factors. Participation in the interventions and NRT use were tested by descriptive statistics. Next, CA and 7-day PPA rates at $\mathrm{T}_{1}$ were assessed for each group by conducting $\chi^{2}$ tests (a) for an intention-to-treat scenario using all cases in which those patients who were lost to follow-up were treated as smokers, and (b) for an observed scenario in which only those patients who fully completed $T_{0}$ and $T_{1}$ were included. The above named analyses were applied to the total available dataset by including patients for relevant analyses although they had one or more missing values on important variables (pairwise deletion).

Because raw abstinence rates do not provide accurate insight into the effects of the TC and FC intervention on CA since they are unadjusted for the multi-level structure of the data, baseline differences, possible moderators and relevant covariates, advanced statistical analysis for CA were done and regarded as the primary outcome. The patients in this study were nested within hospital, resulting in a potential violation of the assumption of independence of observations. To test the existence of hospital-level variation, a multi-level approach was applied (Maas \& Hox, 2005), specifically a generalized linear mixed model (GLMM) which allowed the prediction of CA based on several predictors and accounted for the correlated data structure. The GLMM logistic regression analyses tested the effect of the interventions (UC as reference group), controlling for time-effects, baseline differences between the three groups, predictors of attrition, and a range of demographic, smoking-related and social-cognitive factors in order to correct for possibly confounding factors, and to reduce unexplained variance, thus increasing the power of the tests. Covariates were included in the models due to their clinical significance for smoking cessation in cardiac patients. Interaction effects (intrinsic motivation, intention to quit, SES $\times$ group assignment) were additionally tested to better understand the conditions under which the relationship between the interventions and CA existed. A hierarchical top-down elimination procedure was used to assess the contribution of each predictor variable to the outcome. To obtain a parsimonious model, still giving an adequate description of the data, non-significant variables were removed from the model one by one $(p<0.05)$ until 
only variables remained which significantly contributed to the prediction of CA. Due to their importance, group assignment and time-effects were included in all models. To test conditional effects of the interventions for intrinsic motivation and intention to (remain) quit-both assessing a state of motivation to quit smoking -, their continuous scale scores were transformed into $\mathrm{z}$-scores. SES was dichotomized on the basis of its median score which allowed a reasonable comparison between low and high SES groups. When an interaction showed a $p$ value $\leq 0.10$, GLMM analyses were repeated for subgroups based on the moderator, changing the reference category in categorical variables or changing the value of the moderator in continuous variables to be able to make pairwise comparisons (Twisk, 2006).

For the prediction of CA, we performed two GLMM analyses: (a) for the intention-to-treat scenario (primary outcome analysis), and (b) for the observed scenario (secondary outcome analysis). For all GLMM analyses, patients were excluded from analysis if any predictor variable was missing (listwise deletion). Statistical significance was set at $\alpha=0.05$.

\section{Results}

Table 1 shows that the three groups were similar in important demographic and cardiac risk factors. Patients were predominantly men, and most patients had an admission diagnosis of ACS. Regarding smoking history, nicotine dependence was significantly higher in the FC group compared to the UC group, and patients in the UC group reported significantly more quit attempts in the past 12 months than patients in the TC group. Intrinsic motivation to quit smoking was significantly higher in TC and FC group compared to the UC group.

The analysis of attrition at 6-month follow-up revealed that there was no difference in the likelihood of being lost to follow-up between the three groups. However, patients lost to follow-up were more often women $(O R=0.61$, $95 \%$ CI $0.38,0.97, p=0.04)$, were older $(O R=1.03$, $95 \%$ CI $1.00,1.05, p=0.03$ ), and were more likely to have made a quit attempt in the past $(O R=1.57,95 \% \mathrm{CI}$ $1.00,2.45, p=0.05)$ than patients who remained in the study.

\section{Intervention participation}

According to the telephone counselors and face-to-face counselors, almost all the patients assigned to the experimental groups received the out-patient interventions. On average, $90.5 \%$ of the patients in the TC group received the intervention (at least one session) while they had an average of $4.89( \pm 2.7)$ calls. According to nurses registration forms at $\mathrm{T}_{0}, 78.3 \%$ of the eligible patients in the TC group $(n=137)$ received nicotine patches, that they used on average for $5.5( \pm 3.2)$ weeks. Of the patients in the FC group, $96.8 \%$ received the outpatient intervention (at least one session) and had an average of $5.02( \pm 2.22)$ sessions. Comparably to patients in the TC group, $80.6 \%$ of the eligible patients in the FC group $(n=100)$ received patches upon admission, that they used on average for 5.9 $( \pm 4.1)$ weeks (not in a Table).

Smoking abstinence rates at $T_{1}$

Table 2 depicts unadjusted self-reported smoking abstinence rates by group at 6-month follow-up. In the intention-to-treat scenario, $31.5 \%$ of the patients in the UC group achieved CA compared to $42.2 \%$ in the TC and $40.6 \%$ in the FC group. For 7-day PPA, abstinence rates were about $3 \%$ higher for the three groups. Pairwise $\chi^{2}$ analysis revealed significant differences for the TC group compared to the UC group for CA $\left(\chi^{2}(1)=5.59\right.$, $p=0.02)$ and 7-day PPA $\left(\chi^{2}(1)=5.20, p=0.02\right)$. The FC group only differed marginally from the UC group for CA $\left(\chi^{2}(1)=3.44, \quad p=0.06\right)$ and 7-day PPA $\left(\chi^{2}(1)=3.04, p=0.08\right)$. The TC and FC group did not differ for either form of abstinence (CA: $\chi^{2}(1)=0.09$, $p=0.76$; 7-day PPA: $\chi^{2}(1)=0.11, p=0.74$ ).

Regarding the observed scenario, $37.8 \%$ of the patients in the UC group achieved CA compared to $54.1 \%$ in the TC and $51.2 \%$ in the FC group. Abstinence rates were about $3 \%$ higher considering 7-day PPA as the outcome. Pairwise $\chi^{2}$ analysis showed that the TC group differed significantly on both CA $\left(\chi^{2}(1)=9.83, p=0.00\right)$ and 7-day PPA $\left(\chi^{2}(1)=9.70, p=0.00\right)$ from the UC group. Moreover, the FC group differed significantly from the UC group for CA $\left(\chi^{2}(1)=5.59, p=0.02\right)$ and 7-day PPA $\left(\chi^{2}(1)=5.25, p=0.02\right)$. The TC and FC group did not differ in their abstinence rates (CA: $\chi^{2}(1)=0.24$, $p=0.62$; 7-day PPA: $\chi^{2}(1)=0.29, p=0.59$ ).

Conditional effects of the interventions on smoking abstinence at $\mathrm{T}_{1}$ : intention-to-treat scenario

The goal of the main analysis was to estimate the effect of the interventions on CA in the intention-to-treat scenario, and whether that effect was moderated by patients' intrinsic motivation to quit, intention to (remain) quit, and SES, controlled for a set of covariates. Although intraclass correlations were small and not statistically different from zero, we applied a GLMM for each analysis to be able to account for hospital effects. 
Table 1 Characteristics of the total sample $(n=625)^{\mathrm{a}}$ and comparison of the UC $(\mathrm{n}=245)$, TC $(\mathrm{n}=223)$ and FC ( $\left.\mathrm{n}=157\right)$ groups at baseline

\begin{tabular}{|c|c|c|c|c|c|c|}
\hline Variables & Total & UC & TC & FC & $\chi^{2} / F$ ratio & $p$ \\
\hline Age, $M(S D)$ & $55.92(10.71)$ & $56.09(10.97)$ & $55.31(10.53)$ & $56.54(10.54)$ & 0.65 & 0.522 \\
\hline Sex (male), $n(\%)$ & $457(73.1)$ & $183(74.7)$ & $163(73.1)$ & $111(70.7)$ & 0.77 & 0.678 \\
\hline \multicolumn{7}{|l|}{ Marital status, $n(\%)$} \\
\hline Married with/without children & $414(67.8)$ & $161(67.6)$ & $151(68.9)$ & $102(66.2)$ & \multirow[t]{2}{*}{0.31} & \multirow[t]{2}{*}{0.857} \\
\hline Single/divorced/widow & $196(32.1)$ & $76(32.1)$ & $68(31.1)$ & $52(33.8)$ & & \\
\hline \multicolumn{7}{|l|}{ Education level $^{\mathrm{b}}, n(\%)$} \\
\hline Low education & 247 (40.4) & $99(41.8)$ & $84(38.2)$ & $64(41.6)$ & \multirow[t]{3}{*}{2.37} & \multirow[t]{3}{*}{0.667} \\
\hline Intermediate education & $236(38.6)$ & $85(35.9)$ & $88(40.0)$ & $63(40.9)$ & & \\
\hline High education & $128(20.9)$ & $53(22.4)$ & $48(21.8)$ & $27(17.5)$ & & \\
\hline \multicolumn{7}{|l|}{ Disease diagnosis, $n(\%)$} \\
\hline $\mathrm{ACS}^{\mathrm{c}}$ & $535(85.7)$ & $212(86.9)$ & $192(86.1)$ & $131(83.4)$ & \multirow[t]{3}{*}{5.18} & \multirow[t]{3}{*}{0.270} \\
\hline Stable angina & $53(8.5)$ & $16(6.6)$ & $23(10.3)$ & $14(8.9)$ & & \\
\hline Other/unspecified diagnosis & $36(5.8)$ & $16(6.6)$ & $8(3.6)$ & $12(7.6)$ & & \\
\hline Previous hospital admission, $n(\%)$ & $124(20.1)$ & $49(20.4)$ & $37(16.7)$ & $38(24.5)$ & 3.45 & 0.179 \\
\hline Nicotine dependence $^{\mathrm{d}}, M(S D)$ & $5.32(2.18)$ & $5.09(2.33)$ & $5.31(2.10)$ & $5.69(2.00)$ & 3.53 & $0.030 *$ \\
\hline Average cigarettes per day, $M(S D)$ & $20.84(12.06)$ & $19.71(10.43)$ & $21.13(13.79)$ & $22.19(11.69)$ & 2.12 & 0.123 \\
\hline 7-day PPA at admission, $n(\%)$ & $201(32.5)$ & $89(36.8)$ & $66(29.9)$ & $46(29.7)$ & 3.28 & 0.194 \\
\hline Quit attempt past 12 months, $n(\%)$ & $189(30.7)$ & $87(36.6)$ & $56(25.2)$ & $46(29.7)$ & 7.03 & $0.030 *$ \\
\hline HADS-anxiety $^{\mathrm{e}}, M(S D)$ & $6.50(4.13)$ & $6.09(4.06)$ & $6.70(4.18)$ & $6.84(4.14)$ & 1.97 & 0.140 \\
\hline HADS-Depression $^{\mathrm{f}}, M(S D)$ & $5.42(4.09)$ & $5.55(4.08)$ & $5.22(4.10)$ & $5.51(4.10)$ & 0.43 & 0.652 \\
\hline Self-efficacy to smoking abstinence ${ }^{\mathrm{g}}, M(S D)$ & $2.68(0.81)$ & $2.69(0.89)$ & $2.75(0.72)$ & $2.57(0.78)$ & $2.24^{\dagger}$ & 0.108 \\
\hline Smoking partner, $n(\%)$ & $235(38.0)$ & $100(41.3)$ & $81(36.5)$ & $54(34.8)$ & 2.21 & 0.697 \\
\hline Intrinsic motivation to quit ${ }^{\mathrm{h}}, M(S D)$ & $3.45(0.67)$ & $3.36(0.77)$ & $3.51(0.59)$ & $3.52(0.60)$ & 4.15 & $0.016^{*}$ \\
\hline Intention to (remain) quit ${ }^{\mathrm{i}}, M(S D)$ & $7.49(2.19)$ & $7.49(2.34)$ & $7.50(2.13)$ & $7.48(2.02)$ & 0.07 & 0.993 \\
\hline
\end{tabular}

${ }^{a}$ Missing data are excluded (pairwise deletion) so $n<625$ for some analyses

${ }^{\mathrm{b}}$ Low education $=$ primary and basic vocational schools, intermediate education $=$ secondary vocational schools and high school degrees, high education $=$ higher vocational school degrees, college or university degrees

${ }^{c}$ ACS $=$ acute coronary syndrome, includes unstable angina pectoris and (non-)stemi $=($ non-)ST elevation myocardial infarction

${ }^{\mathrm{d}}$ Range from $0=$ low nicotine dependence to $10=$ high nicotine dependence

e Range from $0=$ low anxiety level to $21=$ high anxiety level

${ }^{\mathrm{f}}$ Range from $0=$ low depression level to $21=$ high depression level

g Range from $0=$ low self-efficacy to $4=$ high self-efficacy towards smoking abstinence

${ }^{\text {h }}$ Range from $0=$ low intrinsic motivation to $4=$ high intrinsic motivation to quit smoking

${ }^{\mathrm{i}}$ Range from $2=$ weak intention to $10=$ strong intention to (remain) quit smoking

$* p<0.05$ (significantly different to referent group [usual care])

$\dagger$ For non-equal variances between the groups, the Brown-Forsythe statistic and $p$ value are reported. Post-hoc tests: For nicotine dependence, Tukey post hoc tests reveal that FC differs significantly from UC. For quit attempts over the past 12 months, $\chi^{2}$ analysis reveal that TC and UC differ significantly from each other. For intrinsic motivation to quit, Tukey post hoc tests reveal that TC and FC both differ significantly from UC

The intention-to-treat analysis revealed that neither intrinsic motivation $(p=0.70)$, nor intention to (remain) quit $(p=0.24)$ were significant moderators of the intervention effects for CA. However, the analysis revealed a marginally significant conditional effect of the interventions on CA for SES $(p=0.09)$. The interaction between TC and SES was particularly significant $(p=0.03)$ (Table 3). The final regression model, adjusted for covariates, yielded significant positive conditional effects for both TC and FC compared with UC on CA for patients with lower SES, with a larger effect for TC. Repeating the analysis yielded no conditional effects of the interventions on CA for patients with higher SES; TC and FC did not increase the odds of being CA for higher SES patients when compared to UC (TC: $O R=1.34,95 \%$ CI 0.52 , $3.46, p=0.54$; FC: $O R=1.68,95 \%$ CI $0.67,4.21$, $p=0.27$ ) (not in a Table). The results further revealed that an older age, being diagnosed with ACS, smoking behavior 
Table 2 Smoking abstinence rates at 6-month follow-up

\begin{tabular}{|c|c|c|c|c|c|c|}
\hline & $\%$ CA (1) & $\chi^{2}$ & $p$ & $\%$ 7-day PPA (1) & $\chi^{2}$ & $p$ \\
\hline \multicolumn{7}{|c|}{ Intention-to-treat scenario: all available cases with patients lost to follow-up $(n=608)^{\mathrm{a}}$} \\
\hline $\mathrm{UC}(n=235)$ & $31.5(n=74)^{\mathrm{b}}$ & & & $34.5(n=81)^{\mathrm{b}}$ & & \\
\hline $\mathrm{TC}(n=218)$ & $42.2(n=92)^{\mathrm{c}}$ & 6.32 & 0.043 & $45.0(n=98)^{\mathrm{c}}$ & 5.82 & 0.054 \\
\hline \multirow[t]{2}{*}{$\mathrm{FC}(n=155)$} & $40.6(n=63)^{\mathrm{b}, \mathrm{c}}$ & & & $43.2(n=67)^{\mathrm{b}, \mathrm{c}}$ & & \\
\hline & $\%$ CA (2) & $\chi^{2}$ & $p$ & \% 7-day PPA (2) & $\chi^{2}$ & $p$ \\
\hline \multicolumn{7}{|c|}{ Observed scenario: all complete cases without patients lost to follow-up $(n=489)^{\mathrm{a}}$} \\
\hline $\mathrm{UC}(n=196)$ & $37.8(n=74)^{\mathrm{b}}$ & & & $41.3(n=81)^{\mathrm{b}}$ & & \\
\hline $\mathrm{TC}(n=170)$ & $54.1(n=92)^{\mathrm{c}}$ & 11.06 & 0.004 & $57.6(n=98)^{\mathrm{c}}$ & 10.84 & 0.004 \\
\hline $\mathrm{FC}(n=123)$ & $51.2(n=63)^{\mathrm{c}}$ & & & $54.5(n=67)^{\mathrm{c}}$ & & \\
\hline
\end{tabular}

at admission and a strong intention to (remain) quit at admission significantly increased the likelihood of CA in the intention-to-treat scenario. On the other hand, factors related with a decreased likelihood of CA were higher having made a previous quit attempt and a high depression level at $\mathrm{T}_{0}$. Time effects were included in the model as statistical controls, but these were overall insignificantly related to $\mathrm{CA}(F(5)=1.28, p=0.27)$.

In order to assess the difference in effect for low versus high SES patients, additional post hoc $\chi^{2}$ tests were conducted analyzing $\mathrm{CA}$ rates at $\mathrm{T}_{1}$ for each group for low versus high SES patients. By selecting low SES patients, the UC group had $26.0 \%$ quitters, versus $42.7 \%$ quitters in the TC group and $38.9 \%$ quitters in the FC group $\left(\chi^{2}(2)=8.67, p=0.01\right)$. When only high SES patients were included, the UC group had $42.1 \%$ quitters, versus $41.7 \%$ quitters in the TC group and $43.5 \%$ quitters in the FC group, respectively $\left(\chi^{2}(2)=0.06, p=0.97\right)$.

Effects of the interventions on smoking abstinence at $\mathrm{T}_{1}$ : observed scenario

Table 4 shows the GLMM regression models for CA in the observed scenario. In this secondary analysis, no significant interaction effects for intrinsic motivation $(p=0.88)$, intention to (remain) quit $(p=0.42)$ and $\operatorname{SES}(p=0.21)$ and were found. The final regression model (non-significant interaction terms excluded) adjusted for covariates, however, revealed significant effects for TC and FC on $\mathrm{CA}$, both compared with UC, with a stronger effect for TC. Furthermore, an older age, smoking behavior at admission and a strong intention to (remain) quit at admission significantly increased the likelihood of CA in the observed scenario. On the other hand, factors related with a decreased odds of CA were having made a previous quit attempt, having a partner who smoked and a high level of depression at admission. Time of the patients' inclusion to the study revealed no significant effect on CA $(F(5)=$ $1.43, p=0.21$ ) in the observed scenario.

\section{Discussion}

Given the importance of smoking cessation in cardiac patients, this study tested the effectiveness of two intensive counseling methods combined with NRT in terms of smoking abstinence six months after patients' hospitalization for CHD. To our knowledge, no previous studies compared a telephone delivered and a face-to-face delivered counseling intervention supplemented with NRT in this patient group, nor tested which patient characteristics might moderate the effect of the interventions on smoking abstinence.

Our study yields several important findings. First, those patients who received an intervention were more likely to be abstinent from smoking six months after hospital discharge than those who received usual smoking cessation care. The TC and FC intervention resulted in similar absolute increases of smoking abstinence rates. Secondly, when adjusting for relevant covariates and including patients lost to followup, SES moderated the effect of the interventions on smoking abstinence. Participation in the interventions did not increase the odds of smoking abstinence compared to UC for higher SES patients, whereas for lower SES patients the conditional odds of smoking abstinence were three to four times greater in TC and FC group compared to the UC group, with somewhat higher odds for TC than FC. Thirdly, TC and FC significantly increased the likelihood of smoking abstinence, also considering those patients who successfully accomplished the 6-month follow-up measurement. Other factors found to be associated with higher odds of smoking abstinence were an older age, being diagnosed with ACS, 
Table 3 Conditional effects of the TC and FC intervention on continued abstinence from smoking for low SES groups at 6-month follow-up for the intention-to-treat scenario $(n=608)$

\begin{tabular}{|c|c|c|c|c|c|c|}
\hline \multirow[t]{2}{*}{ Variables } & \multicolumn{3}{|c|}{ FIRST MODEL $(n=567)$} & \multicolumn{3}{|c|}{ FINAL MODEL $(n=582)^{*}$} \\
\hline & OR & $95 \% \mathrm{CI}$ & $p$ & OR & $95 \%$ CI & $p$ \\
\hline TC & 3.64 & {$[1.36,9.72]$} & 0.010 & 3.40 & {$[1.34,8.65]$} & 0.010 \\
\hline $\mathrm{FC}$ & 3.26 & {$[1.28,8.29]$} & 0.013 & 2.66 & {$[1.10,6.40]$} & 0.029 \\
\hline Age & 1.04 & {$[1.02,1.06]$} & 0.000 & 1.04 & {$[1.02,1.06]$} & 0.000 \\
\hline Sex (male) & 1.47 & {$[0.94,2.30]$} & 0.093 & & & \\
\hline SES (high education) & 2.18 & {$[1.12,4.22]$} & 0.021 & 2.26 & {$[1.20,4.25]$} & 0.011 \\
\hline \multicolumn{7}{|l|}{ Diagnosis } \\
\hline $\mathrm{ACS}^{\mathrm{a}}$ & 2.95 & {$[1.05,8.30]$} & 0.041 & 3.55 & {$[1.26,9.99]$} & 0.016 \\
\hline Unstable angina & 2.02 & {$[0.57,7.08]$} & 0.273 & 2.38 & {$[0.69,8.21]$} & 0.169 \\
\hline Previous admission (no) & 1.24 & {$[0.74,2.06]$} & 0.413 & & & \\
\hline Nicotine dependence & 0.92 & {$[0.84,1.01]$} & 0.077 & & & \\
\hline 7-day PPA at admission & 1.60 & {$[1.05,2.42]$} & 0.029 & 1.61 & {$[1.08,2.40]$} & 0.020 \\
\hline Previous quit attempt & 0.46 & {$[0.30,0.72]$} & 0.001 & 0.45 & {$[0.29,0.68]$} & 0.000 \\
\hline Smoking partner & 0.72 & {$[0.48,1.07]$} & 0.100 & & & \\
\hline HADS-anxiety & 1.05 & {$[0.98,1.11]$} & 0.167 & & & \\
\hline HADS-depression & 0.93 & {$[0.87,0.99]$} & 0.019 & 0.93 & {$[0.89,0.98]$} & 0.004 \\
\hline Self-efficacy & 1.38 & {$[1.04,1.84]$} & 0.028 & & & \\
\hline Intrinsic motivation & 0.95 & {$[0.68,1.34]$} & 0.785 & & & \\
\hline Intention to (remain) quit & 1.14 & {$[1.02,1.28]$} & 0.023 & 1.22 & {$[1.11,1.34]$} & 0.000 \\
\hline $\mathrm{SES} \times$ condition & & $F=2.41$ & 0.091 & & $F=2.18$ & 0.115 \\
\hline High SES $\times$ TC & 0.36 & {$[0.15,0.90]$} & 0.029 & 0.40 & {$[0.17,0.95]$} & 0.037 \\
\hline High SES $\times$ FC & 0.58 & {$[0.21,1.56]$} & 0.276 & 0.63 & {$[0.24,1.65]$} & 0.348 \\
\hline Intraclass correlation (ICC) coefficient & & $\mathrm{ICC}=0.011$ & 0.306 & & $\mathrm{ICC}=0.004$ & 0.410 \\
\hline
\end{tabular}

Sample including patients lost at follow-up as smokers. Respondents with missing data on predictor variables are excluded (listwise deletion) so $n<608$ for the analyses; $n=17$ died and were excluded

Five dummy variables coding time effects are in all models and were entered simultaneously with all other variables, but their coefficients are not reported here

The Model uses reference groups for categorical variables $[$ condition $=$ usual care; time effects $=$ Feb-June 2011; sex $=$ female gender; $\mathrm{SES}=$ high education; diagnosis $=$ non-specified diagnosis; previous admission $=$ yes; 7-day PPA at admission $=$ no; previous quit attempt $=$ no; smoking partner $=$ no]

${ }^{\text {a }}$ ACS acute coronary syndrome, includes unstable angina pectoris and (non-)stemi $=$ (non-)ST elevation myocardial infarction

* Analysis were repeated to the test the outcomes when the interaction term (SES X condition) was excluded. The final model $(n=585)$ revealed significant effects of the TC $(\mathrm{OR}=1.63,95 \% \mathrm{CI} 1.06,2.49, p=0.025)$ and $\mathrm{FC}(\mathrm{OR}=1.66,95 \% \mathrm{CI} 1.03,2.68, p=0.038)$ intervention on continued abstinence. The final model further revealed significant positive effects of age $(O R=1.04,95 \% \mathrm{CI} 1.02,1.06$, $p=0.000)$, an ACS diagnosis $(O R=3.24,95 \%$ CI 1.17, 9.03, $p=0.024)$, 7-day PPA at admission $(O R=1.54,95 \%$ CI $1.04,2.28$, $p=0.033)$, self-efficacy $(O R=1.33,95 \%$ CI 1.02, 1.74, $p=0.038)$ and intention $(O R=1.15,95 \%$ CI 1.04, 1.27, $p=0.008)$, and negative effects of previous quit attempt $(O R=0.46,95 \% \mathrm{CI} 0.31,0.70, p=0.000)$ and depression $(O R=0.94,95 \%$ CI $0.89,0.98, p=0.010)$ on CA

abstinence from smoking at admission and a strong intention (remain) to quit. On the other hand, factors found to impede cardiac patients' abstinence six months after discharge were having made a previous quit attempt, having a partner who smoked, and a high level of depression at the time of hospital admission.

Consistent with our hypothesis and previous findings (Barth et al., 2008; Rigotti et al., 2012), the results of this study show that high intensity counseling that includes extended support for at least one month after discharge is effective in assisting cardiac patients to quit smoking. The finding that TC and FC are comparably effective in increasing quit rates is in line with previous studies in a general population of smokers (Lancaster \& Stead, 2005; Rigotti et al., 2008; Stead et al., 2006). The increase in absolute abstinence rates in our study was somewhat lower compared to the abstinence rates found in the study by Smith and Burgess (Smith \& Burgess, 2009b), which could be explained by the facts that their interventions additionally included a one-hour bedside counseling session and they dealt with considerably lower attrition rates of patients. Yet, our study yielded an interesting conditional 
Table 4 Effects of the TC and FC intervention on continued abstinence from smoking at 6-month follow-up for the observed scenario $(\mathrm{n}=489)$

\begin{tabular}{|c|c|c|c|c|c|c|}
\hline \multirow[t]{2}{*}{ Variables } & \multicolumn{3}{|c|}{ FIRST MODEL $(n=455)$} & \multicolumn{3}{|c|}{ FINAL MODEL $(n=472)$} \\
\hline & OR & $95 \% \mathrm{CI}$ & $p$ & OR & $95 \% \mathrm{CI}$ & $p$ \\
\hline $\mathrm{TC}$ & 3.19 & {$[1.19,8.58]$} & 0.021 & 3.17 & {$[1.22,8.25]$} & 0.018 \\
\hline $\mathrm{FC}$ & 3.21 & {$[1.29,7.99]$} & 0.012 & 2.76 & {$[1.15,6.59]$} & 0.023 \\
\hline Age & 1.03 & {$[1.01,1.06]$} & 0.004 & 1.03 & {$[1.01,1.05]$} & 0.007 \\
\hline Sex (male) & 1.22 & {$[0.74,2.03]$} & 0.434 & & & \\
\hline SES (high education) & 1.37 & {$[0.88,2.13]$} & 0.163 & & & \\
\hline \multicolumn{7}{|l|}{ Diagnosis } \\
\hline $\mathrm{ACS}^{\mathrm{a}}$ & 2.58 & {$[0.82,8.15]$} & 0.107 & & & \\
\hline Unstable angina & 1.57 & {$[0.39,6.28]$} & 0.524 & & & \\
\hline Previous admission (no) & 1.12 & {$[0.63,1.97]$} & 0.703 & & & \\
\hline Nicotine dependence & 0.93 & {$[0.84,1.03]$} & 0.161 & & & \\
\hline 7-day PPA at admission & 1.75 & {$[1.08,2.83]$} & 0.023 & 1.76 & {$[1.12,2.77]$} & 0.015 \\
\hline Previous quit attempt & 0.47 & {$[0.29,0.76]$} & 0.002 & 0.49 & {$[0.31,0.77]$} & 0.002 \\
\hline Smoking partner & 0.61 & {$[0.40,0.95]$} & 0.029 & 0.58 & {$[0.38,0.89]$} & 0.012 \\
\hline HADS-anxiety & 1.05 & {$[0.98,1.13]$} & 0.157 & & & \\
\hline HADS-depression & 0.90 & {$[0.84,0.97]$} & 0.003 & 0.91 & {$[0.87,0.97]$} & 0.001 \\
\hline Self-efficacy & 1.29 & {$[0.93,1.77]$} & 0.126 & & & \\
\hline Intrinsic motivation & 0.92 & {$[0.64,1.34]$} & 0.675 & & & \\
\hline Intention to (remain) quit & 1.20 & {$[1.06,1.36]$} & 0.006 & 1.27 & {$[1.14,1.40]$} & 0.000 \\
\hline Intraclass correlation (ICC) coefficient & & $\mathrm{ICC}=0.019$ & 0.268 & & $\mathrm{ICC}=0.020$ & 0.247 \\
\hline
\end{tabular}

Sample including only patients with follow-up data. Respondents with missing data on predictor variables are excluded (listwise deletion) so $n<489$ for the analyses

Five dummy variables coding time effects are in all models and were entered simultaneously with all other variables, but their coefficients are not reported here. The Model uses reference groups for categorical variables [condition $=$ usual care; time effects $=$ Feb-June 2011; sex $=$ female gender; SES = high education; diagnosis = non-specified diagnosis; previous admission = yes; 7-day PPA at admission = no; previous quit attempt $=$ no; smoking partner $=$ no]

${ }^{\text {a }}$ ACS acute coronary syndrome, includes unstable angina pectoris and (non-)stemi $=($ non-)ST elevation myocardial infarction

effect of the interventions for patients with lower SES, who had a greater likelihood of smoking abstinence when participating in TC or FC compared to UC. We originally expected FC to be more effective in lower SES patients and in less motivated patients, since FC allowed for longer and more in depth sessions than TC, thus the counselors had more time to provide a motivation enhancing intervention. Contrary to our hypothesis, TC and FC were both effective in patients with lower SES, whereas lower SES patients profited somewhat more from TC. One explanation could be that counseling provided by telephone is more appealing to lower SES groups since fewer barriers need to be overcome, contact is largely anonymous and patients do not need to travel to an outpatient smoking cessation clinic. Besides, no costs are involved when participating in $\mathrm{TC}$, whereas the travelling cost and additional time of participating in FC may have been a significant impediment to smoking cessation for patients with lower SES. An earlier study indicated TC to be more convenient to smokers with lower SES than other forms of behavioral counseling (Miller \& Sedivy, 2009). Although TC had shorter counseling time, it is unlikely that the difference in content affected our results, since both counseling parties worked with the same protocol.

Conforming to the findings of previous studies (Thorndike et al., 2008; Dawood et al., 2008; Perez et al., 2008), high levels of depression decreased the odds of smoking abstinence after hospital discharge. Also in line with earlier studies, other factors that were related to a decreased chance of smoking abstinence were having a partner who smoked and failed past quit attempts (e.g., McKenna \& Higgens, 1997). The earlier ENRICHD trial suggested that face-toface cognitive behavioral therapy can be successful to reduce symptoms of depression and increase levels of social support in cardiac patients (Berkman et al., 2003). Although not directly investigated in this study, TC and FC might have reached high effectiveness in those patients who experienced difficulties in quitting after cardiac admission due to motivations to regulate negative emotions through smoking.

Although we expected that patients with a strong motivation to quit smoking profit more from TC than FC and UC, our study did not find a moderating effect of 
cardiac patients' motivation on the effect of the interventions. At hospital admission, the three groups yielded fairly similar scores for their intrinsic motivation and for their intention to (remain) quit, resulting in a relatively homogenous sample with limited variability at baseline. Our hypothesis that patients with higher SES benefit especially from TC was also not confirmed since those with higher SES profited equally from the interventions and UC. An explanation could be that high SES patients were able to quit smoking successfully with little additional help, and thus the interventions provided little additional value. Ultimately, our approach did prove helpful in gaining further insight as to who benefited most from the effects of TC and FC.

An important limitation of this study is the selection bias that may have occurred since the patients were not assigned randomly to the three groups. Although not reported by the nurses, they may have been selective in their recruitment because patients in the intervention groups appeared more motivated in their drive to quit smoking, even though they reported higher nicotine dependence. Selection bias may also have occurred due to patients who refused to participate in the study, probably because they were not interesting in quitting, but ethical constraints did not allow enrollment of patients who declined participation. Second, our design lacked a concurrent control group since all wards started with the implementation of UC, and randomization to the interventions was subsequently conducted at the ward level. Nonetheless, hospital procedures and UC remained constant over the course of the study period, thus it is reasonable to assume that the control group was valid. Third, the refusals of eligible patients were poorly registered by nurses. Thus, accurate data on how many patients were approached for consent and how many consented to participate is lacking, and it is likely that those patients who refused participation differed from those who agreed to participate. Fourth, although intentionto-treat is regarded as the most conservative analysis for smoking cessation outcomes, it might be too conservative for estimating differences in effects between interventions when dealing with high drop-out rates and differential reasons for drop-out between groups. Repeating the attrition analysis per group revealed that having made a previous quit attempt, a higher level of depressive symptoms and having a partner who smoked were significantly related to increased drop-out in the UC group, whereas in the FC group having a smoking partner, and in the TC group an older age were significant predictors for drop-out. However, sample sizes per group were probably too small to reliably address this issue. Nonetheless, it is likely that the true outcome lies somewhere between the observed scenario and the intention-to-treat scenario. The lack of biochemical validation is another drawback, not only because it may inflate abstinence rates, but also because patients in the intervention groups may have felt more embarrassed to report smoking than patients in the UC group, resulting in differential bias of unknown magnitude. Future studies need to biochemically confirm smoking status through cotinine testing. Fifth, the quality of the counseling provided by cardiac nurses trained as professional counselors for this study and experienced telephone counselors of the Dutch Expert Centre for Tobacco Control may have been different, this is thus worth investigating. Last but not least, fewer patients were enrolled in the FC group, which might be related to higher intensity treatment. Intention-to-treat comparisons of FC versus UC might not have differed statistically due to a lack of power in the FC group. This lack of power, but also considering that face-to-face counselors appeared to be newly trained counselors, meaning that they might have had less experience in counseling patients to quit smoking, may have effected our results and explain why FC appeared to be somewhat less effective than TC. Finally, the slight non-adherence to the interventions may have accounted for smaller effect sizes, as also encountered in previous studies (Froelicher et al., 2004; Wiggers et al., 2006; Yerger et al., 2008).

Notwithstanding these limitations, the current study provides evidence that combined approaches of extended smoking cessation counseling and NRT after hospital discharge improve self-reported smoking abstinence rates in patients with CHD. Upon admission, patients should be referred to these interventions as a part of standard care, especially when no systematic smoking cessation interventions are offered. Our study also suggests that high intensity counseling interventions are effective in patients with lower SES, whereas counseling delivered by telephone seems most promising. This has practical implications given that TC is less expensive and likely to generate greater cost-effectiveness than FC, and therefore might be preferred over FC. Future research should use a random allocation of patients and biochemically verify smoking outcomes in order to allow for stronger conclusions about both efficacy and cost-effectiveness of telephone and faceto-face interventions. It is also important to investigate how patients with higher SES may profit from these type of interventions to further improve their abstinence rates.

Acknowledgments This project was supported by a research grant from ZonMw, the Dutch Organization for Health Research and Development (Grant Number: 50-50110-96-524). The authors acknowledge collaboration with the Dutch Expert Centre for Tobacco Control (STIVORO) and collaboration with the hospital offers and cardiac nurses of the Amphia Ziekenhuis (Breda), Atrium Medisch Center (Heerlen), Haga Ziekenhuis (The Hague), Medisch Centrum Haaglanden (Leidschendam), Medisch Centrum Leeuwarden, Onze Lieve Vrouwe Gasthuis (Amsterdam), Twee Steden Ziekenhuis (Tilburg), and VU Medical Center (Amsterdam). Finally, we would 
like to pronounce a particular thanks to all patients for their participation in this study.

Conflict of interest All authors state that they have no conflict of interest to disclose, and no conflict of interest with the Dutch Organization for Health Research and Development that sponsored their research.

\section{References}

Ajzen, I. (2002). Constructing a TpB Questionnaire: Conceptual and methodological considerations. Amherst: University of Massachusetts.

Barth, J., Critchley, J., \& Bengel, J. (2006). Efficacy of psychosocial interventions for smoking cessation in patients with coronary heart disease: A systematic review and meta-analysis. Annals of Behavioral Medicine, 32, 10-20.

Barth, J., Critchley, J., \& Bengel, J. (2008). Psychosocial interventions for smoking cessation in patients with coronary heart disease. Cochrane Database of Systematic Reviews, 1, CD006886.

Benowitz, N. L., \& Gourlay, S. G. (1997). Cardiovascular toxicity of nicotine: Implications for nicotine replacement therapy. Journal of the American College of Cardiology, 29, 1422-1431.

Bentz, C. J., Bayley, K. B., Bonin, K. E., Fleming, L., Hollis, J. F., Hunt, J. S., et al. (2007). Provider feedback to improve 5A's tobacco cessation in primary care: A cluster randomized clinical trial. Nicotine \& Tobacco Research, 9, 341-349.

Berkman, L. F., Blumenthal, J., Burg, M., Carney, R. M., Catellier, D., Cowan, M. J., et al. (2003). Effects of treating depression and low perceived social support on clinical events after myocardial infarction: The enhancing recovery in coronary heart disease patients (ENRICHD) randomized trial. JAMA: Journal of the American Medical Association, 289, 3106-3116.

Berndt, N. C., Bolman, C., de Vries, H., Segaar, D., van Boven, I., \& Lechner, L. (2011). Smoking cessation treatment practices: Recommendations for improved adoption on cardiology wards. The Journal of Cardiovascular Nursing, 28, 35-47.

Berndt, N. C., Bolman, C., Lechner, L., Mudde, A., Verheugt, F. W., \& de Vries, H. (2012a). Effectiveness of two intensive treatment methods for smoking cessation and relapse prevention in patients with coronary heart disease: Study protocol and baseline description. BMC Cardiovascular Disorders, 12, 33. doi: 10.1186/1471-2261-12-33

Berndt, N. C., Bolman, C., Mudde, A. N., Verheugt, F., de Vries, H., \& Lechner, L. (2012b). Risk groups and predictors of short-term smoking abstinence in patients with coronary heart disease. Heart and Lung, 41, 332-343.

Bolman, C., \& de Vries, H. (1998). Psycho-social determinants and motivational phases in smoking behavior of cardiac inpatients. Preventive Medicine, 27, 738-747.

Bolman, C., de Vries, H., \& Mesters, I. (2002a). Factors determining cardiac nurses' intentions to continue using a smoking cessation protocol. Heart and Lung, 31, 15-24.

Bolman, C., de Vries, H., \& van Breukelen, G. (2002b). A minimalcontact intervention for cardiac inpatients: Long-term effects on smoking cessation. Preventive Medicine, 35, 181-192.

Bolman, C., de Vries, H., \& van Breukelen, G. (2002c). Evaluation of a nurse-managed minimal-contact smoking cessation intervention for cardiac inpatients. Health Education Research, 17, 99-116.

Boyle, R. G., Solberg, L. I., Asche, S. E., Boucher, J. L., Pronk, N. P., \& Jensen, C. J. (2005). Offering telephone counseling to smokers using pharmacotherapy. Nicotine \& Tobacco Research, 7, S19S27.
Costa, M. L., Cohen, J. E., Chaiton, M. O., Ip, D., McDonald, P., \& Ferrence, R. (2010). "Hardcore" definitions and their application to a population-based sample of smokers. Nicotine \& Tobacco Research, 12, 860-864.

Critchley, J. A., \& Capewell, S. (2003). Mortality risk reduction associated with smoking cessation in patients with coronary heart disease: A systematic review. JAMA: Journal of the American Medical Association, 290, 86-97.

Dawood, N., Vaccarino, V., Reid, K. J., Spertus, J. A., Hamid, N., \& Parashar, S. (2008). Predictors of smoking cessation after a myocardial infarction: The role of institutional smoking cessation programs in improving success. Archives of Internal Medicine, 168, 1961-1967.

De Vries, H., Mesters, I., Van der Steeg, H., \& Honing, C. (2005). The general public's information needs and perceptions regarding hereditary cancer: An application of the integrated change model. Patient Education and Counseling, 56, 154-165.

Dijkstra, A., de Vries, H., \& Bakker, M. (1996). Pros and cons of quitting, self-efficacy, and the stages of change in smoking cessation. Journal of Consulting and Clinical Psychology, 64, 758-763.

Fernandez, E., Schiaffino, A., Borrell, C., Benach, J., Ariza, C., Ramon, J. M., et al. (2006). Social class, education, and smoking cessation: Long-term follow-up of patients treated at a smoking cessation unit. Nicotine \& Tobacco Research, 8, 29-36.

Fiore, M. C., Jaén, C. R., Baker, T. B., Bailey, W. C., Benowitz, N. L., Curry, S. J., et al. (2008). Treating tobacco use and dependence: 2008 update. U.S. Department of Health and Human Services.

Fishbein, M., \& Ajzen, I. (2010). Predicting and changing behavior: The reasoned action approach. New York: Psychology Press, Taylor \& Francis Group.

Freund, M., Campbell, E., Paul, C., McElduff, P., Walsh, R. A., Sakrouge, R., et al. (2008). Smoking care provision in hospitals: A review of prevalence. Nicotine \& Tobacco Research, 10, 757-774.

Froelicher, E. S., Miller, N. H., Christopherson, D. J., Martin, K., Parker, K. M., Amonetti, M., et al. (2004). High rates of sustained smoking cessation in women hospitalized with cardiovascular disease: The women's initiative for nonsmoking (WINS). Circulation, 109, 587-593.

Gerber, Y., Rosen, L. J., Goldbourt, U., Benyamini, Y., \& Drory, Y. (2009). Smoking status and long-term survival after first acute myocardial infarction a population-based cohort study. Journal of the American College of Cardiology, 54, 2382-2387.

Heatherton, T. F., Kozlowski, L. T., Frecker, R. C., \& Fagerstroem, K. O. (1991). The Fagerstroem test for nicotine dependence: A revision of the Fagerstroem Tolerance Questionnaire. British Journal of Addiction, 86, 1119-1127.

Hennrikus, D. J., Lando, H. A., McCarty, M. C., Klevan, D., Holtan, N., Huebsch, J. A., et al. (2005). The TEAM project: The effectiveness of smoking cessation intervention with hospital patients. Preventive Medicine, 40, 249-258.

Hiscock, R., Judge, K., \& Bauld, L. (2011). Social inequalities in quitting smoking: What factors mediate the relationship between socioeconomic position and smoking cessation? Journal of Public Health, 33, 39-47.

Hoving, E. F., Mudde, A. N., \& de Vries, H. (2006). Smoking and the O pattern; predictors of transitions through the stages of change. Health Education Research, 21, 305-314.

Hughes, J. R., Keely, J. P., Niaura, R. S., Ossip-Klein, D. J., Richmond, R. L., \& Swan, G. E. (2003). Measures of abstinence in clinical trials: Issues and recommendations. Nicotine \& Tobacco Research, 5, 13-25.

Hughes, J. R., Stead, L. F., \& Lancaster, T. (2007). Antidepressants for smoking cessation. Cochrane Database of Systematic Reviews, 1, CD000031. 
Huttunen-Lenz, M., Song, F., \& Poland, F. (2010). Are psychoeducational smoking cessation interventions for coronary heart disease patients effective? Meta-analysis of interventions. British Journal of Health Psychology, 15, 749-777.

Kotz, D., van Litsenburg, W., van Duurling, R., van Schayck, C. P., \& Wesseling, G. J. (2008). Smoking cessation treatment by Dutch respiratory nurses: Reported practice, attitudes and perceived effectiveness. Patient Education and Counseling, $70,40-49$.

Kotz, D., Wagena, E. J., \& Wesseling, G. (2007). Smoking cessation practices of Dutch general practitioners, cardiologists, and lung physicians. Respiratory Medicine, 101, 568-573.

Lancaster, T., \& Stead, L. F. (2005). Individual behavioural counselling for smoking cessation. Cochrane Database of Systematic Reviews, 2, CD001292.

Lichtenstein, E., Glasgow, R. E., Lando, H. A., Ossip-Klein, D. J., \& Boles, S. M. (1996). Telephone counseling for smoking cessation: Rationales and meta- analytic review of evidence. Health Education Research, 11, 243-257.

Maas, C. J. M., \& Hox, J. J. (2005). Sufficient sample sizes for multilevel modeling. Methodology, 1, 86-92.

McKenna, K., \& Higgens, H. (1997). Factors influencing smoking cessation in patients with coronary artery disease. Patient Education and Counseling, 32, 197-205.

Miller, C. L., \& Sedivy, V. (2009). Using a quitline plus low-cost nicotine replacement therapy to help disadvantaged smokers to quit. Tobacco Control, 18, 144-149.

Mohiuddin, S. M., Mooss, A. N., Hunter, C. B., Grollmes, T. L., Cloutier, D. A., \& Hilleman, D. E. (2007). Intensive smoking cessation intervention reduces mortality in high-risk smokers with cardiovascular disease. Chest, 131, 446-452.

Mudde, A. N., Willemsen, M. C., Kremers, S., \& de Vries, H. (2006). Meetinstrumenten voor onderzoek naar roken en stoppen met roken. Den Haag: STIVORO voor een rookvrije toekomst.

Orleans, C. T., Woolf, S. H., Rothemich, S. F., Marks, J. S., \& Isham, G. J. (2006). The top priority: Building a better system for tobacco-cessation counseling. American Journal of Preventive Medicine, 31, 103-106.

Perez, G. H., Nicolau, J. C., Romano, B. W., \& Laranjeira, R. (2008). Depression: A predictor of smoking relapse in a 6-month followup after hospitalization for acute coronary syndrome. European Journal of Cardiovascular Prevention and Rehabilitation, 15, 89-94.

Prochaska, J. O., \& DiClemente, C. C. (1983). Stages and processes of self-change of smoking: Toward an integrative model of change. Journal of Consulting and Clinical Psychology, 51, 390-395.

Ramsay, S. E., Morris, R. W., Whincup, P. H., Papacosta, O., Rumley, A., Lennon, L., et al. (2009). Socioeconomic inequalities in coronary heart disease risk in older age: Contribution of established and novel coronary risk factors. Journal of Thrombosis and Haemostasis, 7, 1779-1786.

Reese, R. J., Conoley, C. W., \& Brossart, D. F. (2006). The attractiveness of telephone counseling: An empirical investigation of client perceptions. Journal of Counseling \& Development, 84, 54-60.

Reid, J. L., Hammond, D., Boudreau, C., Fong, G. T., \& Siahpush, M. (2010). Socioeconomic disparities in quit intentions, quit attempts, and smoking abstinence among smokers in four western countries: Findings from the international tobacco control four country survey. Nicotine \& Tobacco Research, 12, S20-S33.

Reid, R. D., Pipe, A. L., \& Quinlan, B. (2006). Promoting smoking cessation during hospitalization for coronary artery disease. Canadian Journal of Cardiology, 22, 775-780.
Rigotti, N. A. (2009). Helping smokers with cardiac disease to abstain from tobacco after a stay in hospital. Canadian Medical Association Journal, 180, 1283-1284.

Rigotti, N. A., Arnsten, J. H., McKool, K. M., Wood-Reid, K. M., Singer, D. E., \& Pasternak, R. C. (1999). The use of nicotinereplacement therapy by hospitalized smokers. American Journal of Preventive Medicine, 17, 255-259.

Rigotti, N., Clair, C., Munafo, M., \& Stead, L. (2012). Interventions for smoking cessation in hospitalised patients. Cochrane Database of Systematic Reviews, 5, CD001837.

Rigotti, N. A., Munafo, M. R., \& Stead, L. F. (2008). Smoking cessation interventions for hospitalized smokers: A systematic review. Archives of Internal Medicine, 168, 1950-1960.

Ryan, R. M., \& Deci, E. L. (2000). Intrinsic and extrinsic motivations: Classic definitions and new directions. Contempory Educational Psychology, 25, 54-67.

Sarna, L., Bialous, S. A., Wells, M., Kotlerman, J., Wewers, M. E., \& Froelicher, E. S. (2009). Frequency of nurses' smoking cessation interventions: Report from a national survey. Journal of Clinical Nursing, 18, 2066-2077.

Scholte op Reimer, W., De Swart, E., De Bacquer, D., Pyörälä, K., Keil, U., Heidrich, J., et al. (2006). Smoking behaviour in European patients with established coronary heart disease. European Heart Journal, 27, 35-41.

Segaar, D., Bolman, C., Willemsen, M. C., \& de Vries, H. (2006). Determinants of adoption of cognitive behavioral interventions in a hospital setting: Example of a minimal-contact smoking cessation intervention for cardiology wards. Patient Education and Counseling, 61, 262-271.

Segaar, D., Willemsen, M. C., Bolman, C., \& de Vries, H. (2007). Nurse adherence to a minimal-contact smoking cessation intervention on cardiac wards. Research in Nursing \& Health, 30, 429-444.

Siahpush, M., McNeill, A., Borland, R., \& Fong, G. T. (2006). Socioeconomic variations in nicotine dependence, self-efficacy, and intention to quit across four countries: Findings from the International Tobacco Control (ITC) Four Country Survey. Tobacco Control, 15, 71-75.

Smith, P. M., \& Burgess, E. (2009a). Intensive smoking cessation programs for hospitalized coronary patients: A proven intervention in need of implementation. Journal of Clinical Outcomes Management, 16, 396-397.

Smith, P. M., \& Burgess, E. (2009b). Smoking cessation initiated during hospital stay for patients with coronary artery disease: A randomized controlled trial. Canadian Medical Association Journal, 180, 1297-1303.

Stead, L. F., Perera, R., \& Lancaster, T. (2006). Telephone counselling for smoking cessation. Cochrane Database of Systematic Reviews, 3, CD002850.

Taylor, C. B., Houston Miller, N., Killen, J. D., \& DeBusk, R. F. (1990). Smoking cessation after acute myocardial infarction: Effects of a nurse-managed intervention. Annals of Internal Medicine, 113, 118-123.

Thorndike, A. N., Regan, S., McKool, K., Pasternak, R. C., Swartz, S., Torres-Finnerty, N., et al. (2008). Depressive symptoms and smoking cessation after hospitalization for cardiovascular disease. Archives of Internal Medicine, 168, 186-191.

Twisk, J. W. R. (2006). Applied multilevel analysis: A practical guide. Cambridge: Cambridge University Press.

Van Berkel, T. F., Boersma, H., Roos-Hesselink, J. W., Erdman, R. A., \& Simoons, M. L. (1999). Impact of smoking cessation and smoking interventions in patients with coronary heart disease. A systematic review. European Heart Journal, 20, 1773-1782.

Van Berkel, T. F., van der Vlugt, M. J., \& Boersma, H. (2000). Characteristics of smokers and long-term changes in smoking 
behavior in consecutive patients with myocardial infarction. Preventive Medicine, 31, 732-741.

Van Emst, A. J. (2007). Face-to-face counseling to quit smoking: Results of a process- and effectiveness study. The Hague: Dutch Expert Centre for Tobacco Control (STIVORO).

Van Spall, H. G., Chong, A., \& Tu, J. V. (2007). Inpatient smokingcessation counseling and all-cause mortality in patients with acute myocardial infarction. American Heart Journal, 154, 213-220.

Van Stralen, M. M., de Vries, H., Mudde, A. N., Bolman, C., \& Lechner, L. (2011). The long-term efficacy of two computertailored physical activity interventions for older adults: Main effects and mediators. Health Psychology, 30, 442-452.

Van Weel, C., Coebergh, J. W., Drenthen, T., Schippers, G. M., \& Van Spiegel, P. I. (2005). The practice guideline: Treatment of tobacco dependence. Netherlands Journal of Medicine, 149, $17-21$.

Velicer, W. F., \& Prochaska, J. O. (2004). A comparison of four selfreport smoking cessation outcome measures. Addictive Behaviors, 29, 51-60.
West, R., Hajek, P., Stead, L., \& Stapleton, J. (2005). Outcome criteria in smoking cessation trials: Proposal for a common standard. Addiction, 100, 299-303.

Wetta-Hall, R., Ablah, E., Frazier, L. M., Molgaard, C., Berry, M., \& Good, M. (2005). Factors influencing nurses' smoking cessation assessment and counseling practices. Journal of Addictions Nursing, 16, 131-135.

Wiggers, L. C., Smets, E. M., Oort, F. J., Storm-Versloot, M. N., Vermeulen, H., van Loenen, L. B., et al. (2006). Adherence to nicotine replacement patch therapy in cardiovascular patients. International Journal of Behavioral Medicine, 13, 79-88.

World Health Organization. (2007). International statistical classification of diseases and related health problems. 10th revision. Geneva: WHO.

Yerger, V. B., Wertz, M., McGruder, C., Froelicher, E. S., \& Malone, R. E. (2008). Nicotine replacement therapy: Perceptions of African-American smokers seeking to quit. Journal of the National Medical Association, 100, 230-236.

Zigmond, A. S., \& Snaith, R. P. (1983). The hospital anxiety and depression scale. Acta Psychiatrica Scandinavica, 67, 361-370. 